\title{
IMPROVEMENT OF MYOCARDIAL MITOCHONDRIAL FUNCTION AFTER HEMODYNAMIC SUPPORT WITH LEFT VENTRICULAR ASSIST DEVICES IN PATIENTS WITH HEART FAILURE
}

Sun Hi Lee, MD

Nicolai Doliba, $\mathrm{PhD}^{\mathrm{b}}$

Mary Osbakken, $\mathrm{MD}, \mathrm{PhD}^{\mathrm{b}}$

Mehmet Oz, MD

Donna Mancini, $\mathrm{MD}^{\mathrm{c}}$
Objectives: Mitochondrial abnormalities have been described in cardiac tissue of patients with heart failure. These changes may result from chronic hypoxia. Our goal was to determine whether mitochondrial functional capacity can be improved in patients with heart failure by means of long-term left ventricular assist device therapy, which improves myocardial oxygen supply by decreasing myocardial work. Methods: Mitochondria were isolated from myocardial tissue obtained from 13 patients with heart failure without a left ventricular assist device (HF group) and seven patients with heart failure treated with a left ventricular assist device (LVAD-HF group). Mitochondrial respiratory rates (State 2, State 3, and State 4) were measured by means of polarographic techniques with reduced nicotinamide adenine dinucleotide-dependent (pyruvate/malate, $\alpha$-ketoglutarate, glutamate) and -independent (succinate) substrates. The respiratory control index of Chance (State 3/State 4) and Lardy (State 3/State 2) and phosphorus to oxygen ratios were determined. Results: The respiratory control index of Chance was higher in LVAD-HF than in HF when using NADH-dependent substrates pyruvate/malate and $\alpha$-ketoglutarate (pyruvate/malate HF: $4.9 \pm 1.0$; LVAD-HF: $6.5 \pm 1.5$; $\alpha$-ketoglutarate HF: $8.5 \pm$ 2.4; LVAD-HF: $11.8 \pm 2.9$; both $p=0.04$ ). Similarly, the respiratory control index of Lardy was greater in the LVAD-HF than the HF group when $\alpha$-ketoglutarate and glutamate were used as substrates ( $\alpha$-ketoglutarate HF: 7.8 \pm 1.7; LVAD-HF: 9.9 \pm 1.5; glutamate HF: 7.6 \pm 2.2; LVAD-HF: $10.7 \pm 2.1$; both $p=0.04$ ). The phosphorus to oxygen ratio was comparable for both groups using all substrates. No change in mitochondrial respiration was observed after left ventricular assist device therapy with the NADH-independent substrate, succinate. Conclusion: Cardiomyocyte mitochondrial function is improved by long-term therapy with a left ventricular assist device. This improvement suggests that cardiomyocyte metabolic dysfunction in heart failure may be reversed with left ventricular assist device support. (J Thorac Cardiovasc Surg 1998;116:344-9)
$\mathrm{M}$ itochondria are key intracellular organelles that convert oxygen, glucose, free fatty acids, and amino acids into biologically usable energy in the

From the Divisions of Circulatory Physiology a and Cardiothoracic Surgery, ${ }^{\mathrm{c}}$ Columbia Presbyterian Medical Center, New York, N.Y., and the Department of Biochemistry/Biophysics, University of Pennsylvania, ${ }^{\mathrm{b}}$ Philadelphia, Pa.

Received for publication Jan. 27, 1998; revisions requested March 16, 1998; revisions received April 15, 1998; accepted for publication April 22, 1998.

Address for reprints: Donna M. Mancini, MD, Division of Circulatory Physiology, Department of Medicine, Columbia Presbyterian Medical Center, 622 West 168th St., New York, NY 10032.

Copyright (C) 1998 by Mosby, Inc.

$0022-5223 / 98 \$ 5.00+0 \quad \mathbf{1 2 / 1 / 9 1 1 7 5}$ form of adenosine triphosphate (ATP). Control of mitochondrial respiration influences the energy output of the cell and subsequently the organ.

Structural and functional cardiomyocyte mitochondrial abnormalities have been described in animal models of heart failure (HF) and in human HF. ${ }^{1-7}$ Electron micrographs from a canine microembolization model of HF demonstrated structural defects including decreased mitochondria size with matrix depletion and membrane disruption. ${ }^{6}$ Similarly, electron micrographs from hibernating human myocardium demonstrate changes in mitochondrial structure, possibly related to damage from free oxygen radicals. Sabbah and associates ${ }^{6}$ reported extremely low respiratory control indexes (RCIs) in 
Table I. Clinical characteristics

\begin{tabular}{lccc}
\hline & All patients & HF & LVAD-HF \\
\hline No. & 20 & 13 & 7 \\
Age (yr) & $42 \pm 14$ & $43 \pm 14$ & $41 \pm 14$ \\
Gender & & & \\
$\quad$ Women & 7 & 4 & 3 \\
$\quad$ Men & 13 & 9 & 4 \\
Etiology & & & \\
$\quad$ Dilated cardiomyopathy & 14 & 10 & 4 \\
$\quad$ Coronary artery disease & 6 & 3 & 3 \\
Ejection fraction (\%) & $17 \pm 5$ & $16 \pm 4$ & $17 \pm 5$ \\
\hline
\end{tabular}

both animal and human HF when using saponified skinned myocytes. Reduced mitochondrial function has been shown in some animal models of $\mathrm{HF}^{8-10}$ and in human cardiac tissue obtained at the time of transplantation. ${ }^{11}$

Although both functional and structural myocardial mitochondrial defects may contribute to HF, the reversibility of either has not been determined in a clinical population. Implantation of a left ventricular assist device (LVAD) in end-stage HF permits extreme unloading of the left ventricle, thus decreasing myocardial oxygen consumption. Prolonged mechanical unloading in patients with LVADs results in a reversal of neurohormonal stimulation and in a normalization of the compromised pressure/volume relationship in explanted hearts. ${ }^{12-14}$ Histologic improvement, such as the reduction of cellular edema, has also been shown in endomyocardial biopsy tissues after unloading. ${ }^{15}$ However, the effects of prolonged mechanical unloading on myocardial metabolism or mitochondrial function in these patients has not been investigated.

The purpose of the present study was to determine whether cardiomyocyte mitochondrial function is improved after long-term LVAD therapy. Mitochondrial respiratory function was evaluated by means of routine polarographic techniques. ${ }^{16,17}$

\section{Methods}

Patient population. Twenty patients were studied. Eight patients with end-stage $\mathrm{HF}$ had mitochondria isolated from the explanted organ at the time of transplantation and five patients at the time of LVAD implantation. These 13 patients with end-stage HF comprised the HF group. Seven patients with HF had mitochondria isolated at the time of explantation after having been on LVAD support for $185 \pm 81$ days (LVAD-HF; range 35 to 357 days). These seven patients formed the LVAD-HF group. The clinical characteristics of the two groups are presented in Table I. Patient age averaged $42 \pm 14$ years; the majority of patients had dilated cardiomyopathy; ejection fraction averaged 17\% (HF: $16 \pm 4$; LVAD-HF: $17 \pm 5$ ).

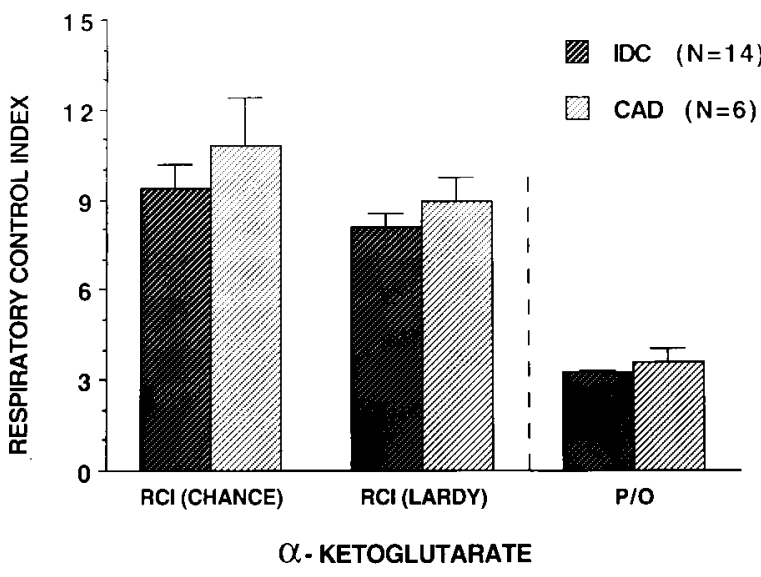

Fig. 1. Comparison of the RCIs of Chance and Lardy and $\mathrm{P} / \mathrm{O}$ ratio using $\alpha$-ketoglutarate as the substrate in patients with coronary artery disease $(C A D)$ and idiopathic dilated cardiomyopathy (IDC).

This study was approved by our institutional review board and consent was obtained from each patient.

Isolation of mitochondria. Approximately $1 \mathrm{gm}$ of myocardium from explanted hearts $(n=15)$ or from the left ventricular apex $(n=5)$ was obtained. For the explanted hearts, usually the left ventricular free wall (or other parts of the myocardium without scar tissue) was used. Right ventricular free wall samples were also obtained in five of the seven patients who had LVADs implanted before transplantation.

Mitochondria were isolated by differential centrifugation according to Kondrashova and Doliba. ${ }^{17}$ Heart tissue was chilled, weighed, and homogenized in a glass PotterElvehjein homogenator (Ann Arbor, Mich.) with a motordriven Teflon pestle. The homogenization buffer contained sucrose $300 \mathrm{mmol} / \mathrm{L}$, HEPES $10 \mathrm{mmol} / \mathrm{L}$, ethylenediaminetetraacetic acid (EDTA) $1 \mathrm{mmol} / \mathrm{L}$, and bovine serum albumin $0.25 \%(\mathrm{pH}=7.4)$. After initial centrifugation ( 7 minutes at $480 \mathrm{~g}$ ), the pellet, containing nuclei and other cellular debris, was discarded. The supernatant was centrifuged at $10,000 \mathrm{~g}$ for 10 minutes to obtain the mitochondrial fraction.

Measurement of mitochondrial oxidative states. Mitochondrial respiratory function (State 2: oxidation with substrate only, no adenosine diphosphate [ADP]; State 3: oxygen consumption in nanoatoms stimulated by ADP; State 4: oxygen consumption in the absence of ADP; RCI; ratio of State 3 to State 4 [Chance]; State 3 to State 2 [Lardy]; and ADP/O: ratio between the nanomoles of ADP phosphorylated to ATP and nanoatoms of oxygen consumed) was measured by means of polarographic methods. ${ }^{18}$ The incubation buffer contained sucrose 250 $\mathrm{mmol} / \mathrm{L}, \mathrm{KCl} 40 \mathrm{mmol} / \mathrm{L}, \mathrm{KH}_{2} \mathrm{PO}_{4} 1 \mathrm{mmol} / \mathrm{L}$, EDTA 0.5 $\mathrm{mmol} / \mathrm{L}$, and HEPES $5 \mathrm{mmol} / \mathrm{L}$ (pH 7.4). Four substrates were used: $\alpha$-ketoglutarate $1 \mathrm{mmol} / \mathrm{L}$; pyruvate plus malate $1 \mathrm{mmol} / \mathrm{L}$; glutamate $1 \mathrm{mmol} / \mathrm{L}$; and succinate 1 $\mathrm{mmol} / \mathrm{L}$. The number of substrates used depended on the availability of adequate cardiac tissue and thus mitochondria. 
Table II. Comparison of end-stage HF and LVAD-HF groups

\begin{tabular}{|c|c|c|c|c|}
\hline Substrate & Pyruvate/malate & $\alpha$-Ketoglutarate & Glutamate & Succinate \\
\hline \multicolumn{5}{|l|}{$\mathrm{HF}$} \\
\hline No. & 13 & 12 & 11 & 11 \\
\hline State $2 *$ & $10.3 \pm 6.5$ & $7.6 \pm 3.8$ & $7.9 \pm 3.6$ & $29.6 \pm 18.0$ \\
\hline State $3^{*}$ & $49.7 \pm 30.4$ & $53.4 \pm 20.7$ & $56.5 \pm 21.2$ & $74 \pm 36$ \\
\hline State $4^{*}$ & $10.2 \pm 5.5$ & $7.3 \pm 3.9$ & $7.1 \pm 3.1$ & $21.8 \pm 11.2$ \\
\hline RCI Chance & $4.9 \pm 1.0$ & $8.5 \pm 2.4$ & $8.1 \pm 2.1$ & $3.6 \pm 1.1$ \\
\hline RCI Lardy & $5.2 \pm 1.3$ & $7.8 \pm 1.7$ & $7.6 \pm 2.2$ & $3.2 \pm 1.1$ \\
\hline $\mathrm{P} / \mathrm{O}$ & $3.3 \pm 0.3$ & $3.2 \pm 0.4$ & $3.3 \pm 0.7$ & $2.3 \pm 0.4$ \\
\hline \multicolumn{5}{|l|}{ LVAD-HF } \\
\hline No. & 7 & 7 & 6 & 6 \\
\hline State $2^{*}$ & $7.5 \pm 4.4$ & $5.5 \pm 3$ & $4.2 \pm 2.4$ & $12.2 \pm 6.4$ \\
\hline State $3^{*}$ & $45.8 \pm 23$ & $51.5 \pm 25$ & $40.4 \pm 19.6$ & $38.6 \pm 27$ \\
\hline State $4^{*}$ & $7.4 \pm 4.4$ & $5.1 \pm 3$ & $4.0 \pm 2.1$ & $10.7 \pm 6.4$ \\
\hline RCI Chance & $6.5 \pm 1.5 \dagger$ & $11.8 \pm 2.9 \dagger$ & $10.7 \pm 2.7$ & $3.3 \pm 0.7$ \\
\hline RCI Lardy & $6.4 \pm 1.5$ & $9.9 \pm 1.5 \dagger$ & $10.7 \pm 2.1 \dagger$ & $2.9 \pm 0.8$ \\
\hline $\mathrm{P} / \mathrm{O}$ & $3.3 \pm 0.3$ & $3.1 \pm 0.2$ & $3.0 \pm 0.2$ & $2.3 \pm 0.5$ \\
\hline
\end{tabular}

*Nanoatoms of oxygen per milligram per minute

$\dagger p<0.05$, HF versus LVAD-HF.

Determination of mitochondrial protein. Mitochondrial protein was determined by means of the Biuret technique $^{18}$ and used to normalize oxidative function data.

Statistical analysis. All results are expressed as mean \pm standard deviation. Comparisons between mean values were performed by the use of a paired Student's $t$ test.

\section{Results}

Mitochondrial function in all patients. The phosphorus to oxygen $(\mathrm{P} / \mathrm{O})$ ratio for all $\mathrm{NADH}$-dependent substrates* averaged 3 and for the NADHindependent substrate, succinate, averaged 2. When mitochondrial data were divided into two subgroups on the basis of the cause of heart failure (coronary artery disease and idiopathic dilated cardiomyopathy), the RCIs of Chance and Lardy were not significantly different. The data using the rate-limiting substrate for the Krebs cycle, $\alpha$-ketoglutarate, are shown in Fig. 1.

Comparison of mitochondrial function of patients in the HF and LVAD-HF groups. Data for HF and LVAD-HF groups are presented in Table II. Statistically significant increases in the RCIs of Chance and Lardy were observed in the LVAD-HF group compared with the end-stage HF group using $\alpha$-ketoglutarate. For the other NADH-dependent substrates (i.e., pyruvate/malate and glutamate) the RCIs of either Chance or Lardy were higher in the LVAD-HF group. In contrast, no change in RCI was observed in the NADH-independent/FAD-de-

* NADH $=$ Reduced nicotinamide adenine dinucleotide pendent substrate* succinate with LVAD therapy. The $\mathrm{P} / \mathrm{O}$ ratios using NADH-dependent or NADHindependent substrates were similar for both groups.

Polarographic tracings from a patient in the HF group and in the LVAD-HF group are shown in Fig. 2. The bold tracing represents the LVAD-HF patient and the thin line demonstrates a typical tracing from a patient with end-stage HF. The slopes of States 2 and 4 are reduced in the LVAD-HF patient relative to the HF subject. This results in higher respiratory control. State 3 is comparable in both $\mathrm{HF}$ and LVAD-HF patients.

Comparison of respiratory control in the right and left ventricles of LVAD-HF patients. In five of the LVAD-HF patients, mitochondria were isolated from both the unsupported right ventricle and the supported left ventricle (Table III). The RCI was similar for both ventricles. This may suggest global improvement in mitochondrial metabolism in both right and left ventricles in the patients supported by LVAD. However, without comparison with mitochondria from the right ventricle of HF patients without LVAD, this cannot be substantiated.

\section{Discussion}

This study demonstrates an improvement of cardiomyocyte mitochondrial function in patients after long-term therapy with LVADs in both right and left ventricular tissue. It also demonstrates that some degree of respiratory control is maintained in hu-

${ }^{*} \mathrm{FAD}=$ Flavin adenine dinucleotide. 
man heart mitochondria after the development of HF.

Mitochondrial function in HF. Whether a bioenergetic defect characterized by an impairment of oxidative phosphorylation is present in the failing human heart is unclear. Prior observations in experimental animal models of HF have demonstrated conflicting results, ${ }^{5-9,19,20}$ with some investigators demonstrating normal RCIs. ${ }^{20}$ In animals in which aortic banding was used to produce HF, the RCI was lower in animals with HF than in control animals. ${ }^{19}$ In rabbits with cardiac hypertrophy produced by an aortic Ameroid constrictor, the RCI was decreased in the failing but not in the hypertrophied hearts. ${ }^{10}$ In these animals, calcium uptake by the mitochondria was significantly decreased in the failing hearts.

Few studies have examined human myocardial mitochondrial function. In 1966, Chidsey and associates $^{21}$ obtained papillary muscle from patients with left ventricular dysfunction who were undergoing valve replacement. They demonstrated a preserved $\mathrm{P} / \mathrm{O}$ ratio and similar $\mathrm{RCIs}$ to those of mitochondria isolated from normal experimental animals and from right ventricular tissue from two patients with tetralogy of Fallot. In 1971, Lindenmayer and colleagues ${ }^{11}$ recorded the RCI from cardiac tissue from heart transplant recipients with coronary artery disease using both NADH-dependent and -independent substrates. They reported a reduced rate of oxygen consumption in the presence of ADP in mitochondria from the failing recipient hearts. More recently, Sabbah and coworkers, ${ }^{6}$ using saponified myocytes from heart transplant recipients, reported a loss of respiratory control in the failing heart. However, the technique of saponization may have damaged mitochondrial membranes and contributed to these findings.

In our study, the $\mathrm{P} / \mathrm{O}$ ratio was normal, consistent with the findings of Lindenmayer, ${ }^{11}$ Chidsey, ${ }^{21}$ and their associates. The RCI tended to be lower than that reported by Lindenmayer and coworkers ${ }^{11}$ and similar to those reported by Chidsey and coworkers. ${ }^{21}$ However, whether the RCI measured in these studies represents a normal rate of respiration in human beings remains unclear, because no historical controls are available for comparison owing to the difficulty in obtaining normal human heart tissue.

In our study, the $\mathrm{RCI}$ and $\mathrm{P} / \mathrm{O}$ ratios in mitochondria of patients with coronary artery disease and dilated cardiomyopathy were similar, suggesting that the end result of these different pathophysiologic processes is similar.

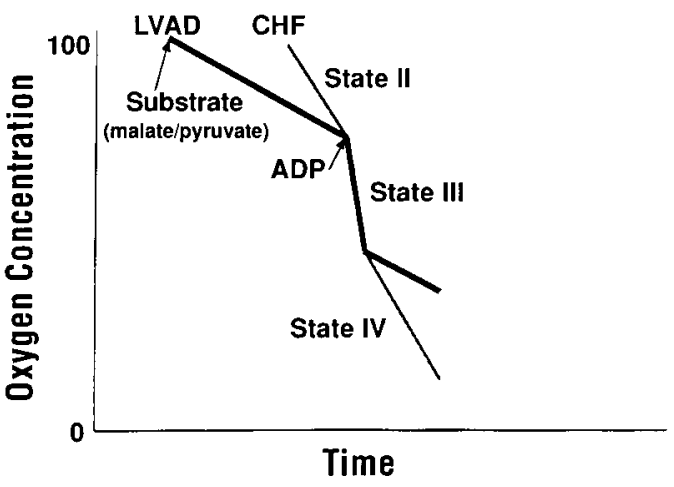

Fig. 2. Oxygen electrode tracing in a patient before and after LVAD support. Oxygen concentration expressed as percent. $C H F$, Congestive heart failure.

Improvement in respiratory control with LVAD therapy. The most significant finding was an improvement in the RCI observed after LVAD support using NADH-dependent Krebs cycle substrates. The improvement in respiratory control resulted primarily from shifts of the slopes of State 2 and State 4. The lack of shift in the slope of State 3 suggests that electron chain transport is unaffected and appears to be normal. The rates of State 2 and 4 respiration are influenced by free fatty acid accumulation and calcium overload, both of which affect membrane potential. These changes decrease the coupling between the oxidation of substrates in the Krebs cycle and ATP synthesis in the respiratory chain. Thus one possible mechanism for the change in the rate of State 4 respiration is an alteration in mitochondrial calcium levels. Cytoplasmic calcium overload could result in increased mitochondrial calcium levels. In the Syrian hamster, the RCI declines as the cardiomyopathic process progresses and is inversely related to the ratio of intracellular calcium and magnesium. ${ }^{22}$ Thus respiratory control diminishes as cytosolic calcium overload occurs. In this hamster model, diminished respiratory control results from altered State 4 respiration, as State 3 respiration and $\mathrm{P} / \mathrm{O}$ ratios remain unchanged. ${ }^{23}$ Whether a similar effect occurs in human HF is unknown. Future studies on isolated mitochondria incorporating calcium measurements may be helpful in clarifying the role of calcium.

Other potential mechanisms for improved mitochondrial function after LVAD therapy may simply be due to structural effects, that is, a reduction in myocyte stretch. Echocardiograms in the patients supported by an LVAD demonstrated markedly 
Table III. Comparison of mitochondrial function in left and right ventricles of LVAD-HF patients $(n=5)$ using $\alpha$-ketoglutarate

\begin{tabular}{lcc}
\hline & Right ventricle & Left ventricle \\
\hline State $2^{*}$ & $8.5 \pm 4$ & $11.9 \pm 3.0$ \\
State $3^{*}$ & $64 \pm 21$ & $66 \pm 15$ \\
State $4^{*}$ & $10.8 \pm 2.7$ & $11.2 \pm 2.7$ \\
P/O ratio & $3.1 \pm 0.1$ & $3.2 \pm 0.2$ \\
\hline
\end{tabular}

*Nanoatoms of oxygen per milligram per minute.

diminished left ventricular size. ${ }^{14}$ Pathology specimens from patients after explantation have also demonstrated reduction in myocyte area. ${ }^{12}$ Alternately, increased oxygen delivery may be a primary factor for the observed changes either by decreasing the diffusion distance as a consequence of myocyte atrophy or increasing supply by virtue of the decreased cellular workload. Decrease in catecholamine stimulation with LVAD support is another potential mechanism for the observed improvement in mitochondrial function. ${ }^{12,13,24}$

It is not surprising that no change in mitochondrial respiration was observed when an NADH independent/FAD-dependent substrate (succinate) was used, because this represents a less efficient use of oxygen. This is in contrast to the NADH-dependent substrates (pyruvate/malate, glutamate), which demonstrate more efficient oxygen use for ATP demonstrated by an increase in RCI and decrease in State 4 respiration in the LVAD-HF group.

Whether the improvement in mitochondrial function after LVAD support translates into improvement in mechanical function cannot be answered by this study. Ejection fractions were similar between the two groups at the time of LVAD insertion; however, cellular and mechanical function were not concordantly measured after a period of LVAD support. Recently, using an isolated myocyte preparation, Dipla and cowork$\mathrm{ers}^{25}$ described the contractile properties of myocytes isolated from five patients with LVAD support compared with myocytes from 19 explanted failing hearts. The fractional shortening, time to peak contraction, time to $50 \%$ relaxation, and fractional shortening after exposure to isoproterenol were all significantly improved in the patients supported with an LVAD. These findings would suggest that mechanical function should improve with LVAD support.

Different patients had different levels of inotropic support that will affect myocardial oxygen consump- tion. Only three of the HF patients were not receiving parenteral inotropic support at the time of transplantation. None of the LVAD patients were receiving parenteral inotropic agents. LVAD support should decrease myocardial oxygen consumption by decreasing myocardial work. However, the measurement of the RCI describes the efficiency of mitochondrial metabolism independent of load. Past evidence from animal and human studies suggest that inotropic agents improve rather than worsen mitochondrial function. Unverferth and associates $^{26}$ described a morphologic improvement in mitochondria from patients with $\mathrm{HF}$ after inotropic support. Quantitative ultrastructural analysis of electron micrographs from biopsy tissue of patients before and after 72-hour infusion of dobutamine showed an increase in cristae-to-matrix ratio and mitochondrial size in comparison with samples derived from patients who received only bedrest. These morphologic improvements do not necessarily mean that there is a coexistent functional improvement. However, animal studies would support both morphologic and functional improvement after treatment with inotropic agents. The effect of dopamine, dobutamine, and amrinone on mitochondrial function in a canine infarct model of cardiogenic shock was studied by Mukae and colleagues. ${ }^{27}$ All of the inotropic agents were shown to significantly improve the RCI in animals receiving succinate or glutamic acid/malic acid compared with animals receiving only saline solution. Morphologic deterioration of mitochondria with swelling and fusion was observed only in the saline-treated animals. Other animal studies using different models have also demonstrated improvement of mitochondrial function with inotropic support. ${ }^{28,29}$ It is therefore unlikely that our findings represent an epiphenomena. If all the patients with end-stage HF (LVAD, nonLVAD) had the same positive inotropic treatment, it is likely that the difference in mitochondrial efficiency (estimated by the RCI) between the two groups would have been exaggerated.

Study limitations. The biochemical techniques used in this study to measure mitochondrial respiration are well established. The accuracy of our protein determinations may be limited by small amounts of tissue. As the tissue was processed immediately, potential damage from prolonged inadequate tissue preservation was not a factor. This study is also limited by the lack of measurements from normal human myocardium. Biopsy specimens do not provide adequate tissue to isolate mitochondria. 
Clinical limitations of this study include the small sample size. Longitudinal rather than cross-sectional analysis would have been preferable. Owing to the prolonged LVAD support in many of these patients, longitudinal analysis was not possible. Additional information including measurement of mitochondrial enzymes, free fatty acids, $\mathrm{pH}$, and ultrastructural assessment of the mitochondria would have helped to elucidate the mechanism of the improved mitochondrial function. Unfortunately, these measurements were not obtained.

\section{Conclusion}

In conclusion, long-term LVAD support in patients with HF reduces cardiovascular load and improves myocardial mitochondrial function. Patients have more efficient energy production after LVAD support. This improvement in myocardial mitochondrial function has important clinical implications, because it suggests some reversibility of cardiac metabolic dysfunction with LVAD support.

\section{REFERENCES}

1. Williams RS. Cardiac involvement in mitochondrial diseases, and vice versa. Circulation 1995;91:1266-8.

2. Anan R, Nakagawa M, Miyata M, et al. Cardiac involvement in mitochondrial diseases: a study on 17 patients with documented mitochondrial DNA defects. Circulation 1995;91: 955-61.

3. Corral Debrinski M, Stepien G, Shoffner J, Lott M, Kanter K, Wallace D. Hypoxemia is associated with mitochondrial DNA damage and gene induction: implications for cardiac disease. JAMA 1991;266:1812-8.

4. Marin-Garcia J, Goldenthal M. Cardiomyopathy and abnormal mitochondrial function. Cardiovasc Res 1994;28:456-63.

5. Ozawa T. Mitochondrial cardiomyopathy. Herz 1994;19:105-18.

6. Sabbah H, Sharov V, Riddle J, Kono T, Lesch M, Goldstein $\mathrm{S}$. Mitochondrial abnormalities in myocardium of dogs with chronic heart failure. J Mol Cell Cardiol 1992;24:1333-67.

7. Shoffner J, Wallace D. Heart disease and mitochondrial DNA mutations. Heart Dis Stroke 1992:1:235-41.

8. Proscheck L, Jasmin G. Hereditary polymyopathy and cardiomyopathy in the Syrian hamster. II. Development of heart necrotic changes in relation to defective mitochondrial function. Muscle Nerve 1982;5:26-32.

9. Lindenmayer GE, Sordahl LA, Schwartz A. Reevaluation of oxidative phosphorylation in cardiac mitochondria from normal animals and animals in heart failure. Circ Res 1968;23: 439-50.

10. Sordahl LA, McCollum WB, Wood WG, Schwartz A. Mitochondria and sarcoplasmic reticular function cardiac hypertrophy and failure. Am J Physiol 1973;224:497-502.

11. Lindenmayer GE, Sordahl LA, Harigaya S, Allen JC, Besch HR Jr, Schwartz A. Some biochemical studies on subcellular systems isolated from fresh recipient human cardiac tissue obtained during transplantation. Am J Cardiol 1971;27:277-83.
12. Levin $\mathrm{H}$, Chen $\mathrm{J}, \mathrm{Oz} \mathrm{M}$, et al. Potential of left ventricular assist devices as outpatient therapy while awaiting transplantation. Ann Thorac Surg 1994;58:1515-20.

13. Estrada-Quintero T, Uretsky B, Murali S, Griffith B, Kormos R. Neurohormonal activation and exercise function in patients with severe heart failure and patients with left ventricular assist system. Chest 1995;107:1499-503.

14. Levin H, Oz M, Chen J, Packer M, Rose E, Burkhoff D. Reversal of chronic ventricular dilation in patients with end stage cardiomyopathy by prolonged mechanical unloading. Circulation 1995;91:2717-20.

15. Fisher P, DeRosa C, Choudri A, Oz M. Mechanical assist device placement in end stage heart failure results in significant ventricular remodeling [abstract]. J Heart Lung Transplant 1997;16:81A.

16. Chance B, Williams GR. The respiratory chain and oxidative phosphorylation. Adv Enzymol 1956;17:65-134.

17. Kondrashova M, Doliba N. Polarographic observation of substrate-level phosphorylation and its stimulation by acetylcholine. FEBS Lett 1984;243:153-5.

18. Gornall A, Bardawill C, David M. J Biol Chem 1949;177: 751-5.

19. Sobel BE, Spann JF, Pool PE, Sonnenblick EH, Braunwald D. Normal oxidative phosphorylation in mitochondria from the failing heart. Circ Res 1967;21:355.

20. Cooper G IV, Puga F, Zujko KT, Harrison CE, Coleman HN III. Normal myocardial function and energetics in volumeoverloaded hypertrophy in the cat. Circ Res 1973;32:140.

21. Chidsey C, Weinbach E, Pool P, Morrow A. Biochemical studies of energy production in the failing human heart. J Clin Invest 1966;45:40-50.

22. Jasmin G, Proschek L. Calcium and myocardial cell injury-an appraisal in the cardiomyopathic hamster. Can J Physiol Pharmacol 1984; 61:891-8.

23. Doliba NM. Calcium as the mediator of stress induced dysfunction in cardiomyopathic hamster hearts? Exp Biol April 1996, Washington DC. Abstract.

24. Khan T, Levin H, Oz M, Katz S. Delayed reversal of impaired metabolic vasodilation in patients with end stage heart failure during long term circulatory support with a left ventricular assist device. J Heart Lung Transplant 1997;16:449-53.

25. Dipla K, Mattiello J, Jeevanandum V, Paolone A, Margulies $\mathrm{K}$, Houser S. Improved myocyte function following mechanical support of the left ventricle in humans with severe heart failure [abstract]. Circulation 1997;96:298A.

26. Unverferth D, Leier C, Magorien R, Croskery R, Svirbely J, Kolibash A, et al. Improvement of human myocardial mitochondria after dobutamine: a quantitative ultrastructural study. J Pharmacol Exp Ther 1980;215:527-32.

27. Mukae S, Yanagishita T, Geshi E, Umetsu K, Tomita M, Itoh $\mathrm{S}$, et al. The effects of dopamine, dobutamine, and amrinone on mitochondrial function in cardiogenic shock. Jpn Heart J 1997;38:515-29.

28. Das A, Harris D. Control of mitochondrial ATP synthase in heart cells: inactive to active transitions caused by beating or positive inotropic agents. Cardiovasc Res 1990;24:411-17.

29. McCormack J, Denton R. The role of calcium in the regulation of intramitochondrial energy production in heart. Biomed Biochim Acta 1987;46:S487-92. 